BNL- 64530

CAP-189-MISC-97C

PARAMETRIC X-RAY RADIATION AS SOURCE OF PULSED, POLARIZED, MONOCHROMATIC, TUNABLE X-RAY BEAM.

\author{
z. Parsa \\ Department of Physics \\ Brookhaven National Laboratory \\ Upton, NY 11973 \\ and \\ A.V. Shchagin \\ Kharkov Institute of Physics and Technology \\ Kharkov 310108, Ukraine
}

June 1997 


\title{
Parametric X-ray Radiation as Source of Pulsed, Polarized, Monochromatic, Tunable X-ray Beam
}

\author{
Z. Parsa ${ }^{\dagger 1}$ and A.V. Shchagin ${ }^{\ddagger 2}$ \\ ${ }^{\dagger}$ Brookhaven National Laboratory \\ 901A Physics Dept., Upton, NY 111973-5000, USA \\ ${ }^{\ddagger}$ Kharkov Institute of Physics and Technology, \\ Kharkov 310108, Ukraine
}

\begin{abstract}
The parametric X-ray radiation (PXR) is a new type of monochromatic, polarized, directed, tunable radiation arising in the vicinity of Bragg directions of a crystal when relativistic particles are passing through the crystal. For the last ten years the PXR properties have been intensively investigated experimentally at moderate incident electron current. In this paper we consider main properties of PXR as a new source of a powerful monochromatic X-ray beam, excited by short high-current electron bunch in a single crystal. It is shown, that using a PXR, one can obtain X-ray beam power of about MW/sr during a short period of time at moderate incident electron energy. Experimental setup for generating pulsed PXR beam is suggested, and possibilities for application of pulsed polarized monochromatic X-ray beams are discussed.
\end{abstract}

\section{INTRODUCTION}

In recent years the interest for creation of new generation of powerful pulsed $\mathrm{X}$-ray source is increasing. Considerations and investigations of different methods of X-ray production for this purpose are in progress. Especially of interest is creation of powerful source of polarized X-ray beam. In this paper we will present and analyze properties of Parametric X-ray Radiation (PXR) as source of powerful pulsed polarized monochromatic X-ray beam.

PXR is coherent type of radiation and it is due to the interaction of electromagnetic field of incident particle with electron subsystem of crystal. From the theory [2] it may be seen that the PXR angular distribution has a sharp conical maximum (reflection) with the gap in the middle, found around the Bragg

1) Supported in part by US Department of Energy Contract No. DE-AC02-76CH00016

2) Supported in part under the Grant of Ukrainian Fund of Basic Research

(C) 1997 American Institute of Physics 
direction. The angular size of the PXR reflection is about or greater than $\frac{1}{\gamma}$, where $\gamma$ is the relativistic factor of incident particle. Earlier experimental investigations have been performed at moderate electron beam current. Here we will consider the case when PXR is excited by high current short pulse of electron beam (bunched electron beam), e.g. as in [17].

The radiation generated by the charged particle moving through a medium with a periodically varying permittivity was first predicted by Fainberg and Khizhnyak [1] in 1957. Later, the radiation of charged particles moving through a crystal in the X-ray band was investigated by Ter-Mikaelian [2] and several versions of theoretical descriptions of PXR were proposed e.g., in Refs. [3-6] . The experimental studies of PXR properties were began in the late 80-ies e.g., in Refs. [7-12], continued in 90-ies e.g., in Refs. [13-15], and are reviewed e.g., in Ref. [16].

\section{FORMULATION}

In this section we will present some of the formulae we have used in our calculations. By analogy with the ordinary diffraction of X-rays in a crystal, there are two schemes for PXR generation: one is the Laue geometry and the other is the Bragg geometry. In both cases, the beam of charged relativistic particles with velocity $\vec{V}$ passes through a crystal-radiator, and the narrow PXR beam (reflection) of a conical shape is generated around the Bragg direction with respect to the crystallographic planes. Most of the experiments were carried out in the Laue geometry. This geometry: is shown in Fig. 1.

The PXR quanta energy may be calculated according to the formula from ref. [2]

$$
E_{C R}=\hbar \omega_{C R}=\frac{c \hbar|\vec{g} \cdot \vec{V}|}{c-\sqrt{\varepsilon_{0}} \vec{V} \cdot \vec{\Omega}},
$$

where $\hbar$ is the Plank constant divided by $2 \pi, \varepsilon_{0}$ is the constant part of the medium permittivity, $\vec{g}$ is the reciprocal lattice vector, $\vec{\Omega}$ is the unit vector in the direction of the detector, $c$ is the light velocity.

The width at half maximum of the PXR spectral peak for a detector of small angular size is

$$
\Delta E_{C R} \approx E_{C R} \frac{\Delta \theta}{\operatorname{tg}(\theta / 2)}
$$

where $\Delta \theta$ is the polar angular resolution of the experiment. The $\Delta \theta$ value should include the detector size and the beam spot size on the target, and also the electron beam divergence in the target (including electron multiple scattering). Formula (2) was derived and confirmed experimentally in ref. [9]. 
The differential yield is described by the formula for the number of quanta, derived in ref. [9] from the kinematical theory [2]

$$
\begin{gathered}
Y_{\vec{g}}=\frac{d N}{n d \Omega}=\frac{e^{2} \omega L\left|\chi_{\vec{g}}(\omega)\right|^{2}}{2 \pi \hbar \varepsilon_{0}^{3} V\left(\frac{c}{\sqrt{\varepsilon_{0}}}-\vec{V} \cdot \vec{\Omega}\right)} . \\
\left|\frac{\left(\omega \sqrt{\varepsilon_{0}} / c\right) \vec{\Omega} \times\left[\left(\omega \varepsilon_{0} / c^{2}\right) \vec{V}+\vec{g}\right]}{\left.\mid\left(\omega \sqrt{\varepsilon_{0}} / c\right) \overrightarrow{\Omega_{\perp}}-\overrightarrow{g_{I}}\right]^{2}+\left(\frac{\omega}{V}\right)^{2}\left[(1 / \gamma)^{2}+\left(\frac{V}{c}\right)^{2}\left(1-\varepsilon_{0}\right)\right]}\right|^{2},
\end{gathered}
$$

where $d N$ is the number of quanta with frequency $\omega=\omega_{C R}$ (see formula (1)) emitted in the solid angle $d \Omega$ as $n$ particles with the charge $e$ and relativistic factor $\gamma$ pass through a crystal of a thickness $L ; \chi_{\vec{g}}(\omega)$ is the Fourier component of the variable part of permittivity; $\overrightarrow{\Omega_{\perp}}, \overrightarrow{g \perp}$ are the components of $\vec{\Omega}$, $\vec{g}$ perpendicular to $\vec{V}$.

The factor $L$ with the attenuation of PXR in target taken into account should be used in the form [11]

$$
L=T_{e}\left|\frac{\vec{t} \cdot \vec{\Omega}}{\vec{t} \cdot \vec{v}}\right|\left[1-\exp \left(-\frac{T}{T_{e}|\vec{t} \cdot \vec{\Omega}|}\right)\right]
$$

where $T_{e}=T_{e}(\omega)$ is the e-fold attenuation length of radiation with frequency! $\omega ; T$ is the target plate thickness; $\vec{t}$ is the unit vector perpendicular to the target plane; $\vec{v}=\frac{\vec{V}}{V}$.

Calculations by formulae (1), (2), (3), (4) have been compared to experimental data on differential PXR yield in refs. $[9,11,12,16]$ and a good agreement. of calculated and measured data was found.

The background conditions in PXR spectra were discussed in ref. [12]. where it was shown that the resulting PXR spectral peak/background ratio in the vicinity of Bragg direction may be about or more than 100 even at a moderate incident electron energy.

The linear polarization directions are oriented radially around the reflection center. If the detector is of the same size or greater than the PXR reflections, the average polarization should be generally close to zero. However, by collimating the corresponding differential part of the PXR reflection, the X-ray beam with an arbitrary direction of linear polarization may be chosen for applications. This was confirmed experimentally in ref. [8]. In particular, if the reciprocal lattice vector $\vec{g}$ is in the detection plane, the linear polarization direction is in the detection plane too (parallel polarization [16]).

So, PXR is perspective source of monochromatic, tunable, polarized X-ray beam with a low spectral background. The mentioned PXR properties have been confirmed at moderate electron beam current for PXR quanta energies $5-400 \mathrm{keV}$ at electron beam energy $15-1200 \mathrm{MeV}[16]$. 


\section{PULSED PXR}

Below we will consider PXR as source of powerful monochromatic polarized $\mathrm{X}$-ray beam, generated in a crystal by short ełectron bunch, when the intensity. of PXR beam will be high enough during a short pulse.

The suggested experimental layout is shown in Fig.1. The beam of accelerated bunched electrons (labeled as 1) passes in vacuum through a target. (labeled as 2). A thin silicon single crystal plate is used as a target 2. The crystallographic plane $(\overline{2} 20)$ is parallel to the plate surface. The target is placed in a 3-axis vacuum goniometer and is prealigned so that the $\langle\overline{2} 20\rangle$ axis should be parallel to the velocity vector $\vec{V}$ of the (beam) electrons, and the crystallographic plane (111) should be perpendicular to the detection plane and parallel to the vector $\vec{V}$. At $\phi=0$ the crystallographic plane (111) is parallel to the electron beam and the reciprocal lattice vector $\vec{g}=\langle 111\rangle$ lie in the detection plane. The PXR reflection (labeled as 3) from planes (111) has an asymmetrical conical form and the Bragg direction (labeled as 4) is the cone axis. To obtain polarized X-ray beam we can use the part of PXR reflection, where PXR yield has maximum value. This part has conical form with full cone angle $1 / \gamma$ (at moderate incident electron energy). The cone axis is at angle $\theta=\theta_{B}+1 / \gamma$. The angle of crystal rotation is $\phi=\theta_{B} / 2$. The direction of linear polarization lies in the plane of the figure. Detector or: consumer (labeled as 5) of pulsed polarized X-ray beam is shown in Fig. 1.

Target thickness is chosen to provide electron beam divergency in the target no more than $1 / \gamma$. Target thickness $T=T_{\gamma}$ can be found from the equation

$$
\frac{1}{\gamma}=\theta_{s}(T)
$$

where $\theta_{s}$ is the electron multiple scattering angle [2] in silicon. Note, that $T_{\gamma}$ is independent of incident electron energy and is a function only of target material properties. For silicon single crystal $T_{\gamma} \approx 60 \mathrm{mkm}$. We will use this thickness in our calculations.

To calculate power of pulsed PXR beam, we used a typical (for an available) parameter set for a bunched electron beam: charge of electron bunch $1 n C$ and bunch duration of $333 \mathrm{fs}$ at electron beam energies 50,100 and $200 \mathrm{MeV}$.

Target temperature is increasing when the target is working as crystalradiator in electron beam. It has been shown in ref. [14], that Si single crystal can work as PXR radiator at average electron beam current of about 0.5 $A$ at electron beam radius of $1 \mathrm{~mm}$. In our case, average bunched electron beam current is function of accelerator repetition rate and repetition rate of real accelerators usually is much less than $0.5 \mathrm{~A} /\left(1.6 \cdot 10^{-9} \mathrm{C}\right) \sim 3 \cdot 10^{8} \frac{\mathrm{bunches}}{\mathrm{s}}$. Therefore we do not need to take into account crystal-radiator heating and we use the room temperature for Si single crystal in our estimations.

In calculations of bunched PXR beam properties we used Eqs. (1), (3) from refs. $[2,9]$, which was confirmed experimentally in previous investigations 
$[9,11,12,16]$ at moderate electron beam current. Results of the calculations of polarized X-ray beam power in units MW/steradian are shown in Fig.2, as a function of required energy of X-ray quanta. These results may be used to estimate the power flux in term of full cone ángle of $\leq 1 / \gamma$. For every chosen quanta energy it is necessary to provide a required setup geometry (angles $\phi, \theta$, see Fig. 1). For the data, represented in Fig. 2, angles $\theta$ are in the range $40-170 \mathrm{mrad}$. The width of PXR spectral line may be easily calculated using formula (2). It is seen from Fig. 2, that intensity of polarized PXR beam is high enough and it may be applied in different fields. Pulsed.PXR with short pulse, high intensity electron beam are discussed in refs. [17-19].

\section{APPLICATIONS OF PULSED PXR BEAM.}

During the last decades numerous applications of a monochromatic X-ray beam have been developed. Now the most popular source of an X-ray beam is synchrotron radiation. But synchrotron radiation usually is not able to provide high power X-ray pulses. As can be seen from Fig.2, PXR is a perspective source of powerful short pulses of polarized, monochromatic tunable X-ray beam. Duration of X-ray pulses is determined by duration of electron beam. bunch. Power of the X-ray pulse is proportional to the charge of electron bunch. Direction of linear polarization of X-ray beam at consumer 5 is in the plane of Fig. 2.

A powerful polarized pulsed X-ray beam, suggested in this paper, may. be applied in different areas of science and technology. Following are some of the applications, where the polarized X-ray beam may be used:

1. For measurements of bunched electron beam parameters

2. For measurements of short-time crystal-radiator properties

3. For medical and biological applications and diagnostics

4. For investigations of femtosecond processes in solid state.

5. For precision calibration of spectral, angular and polarization properties of new generation of astrophysical space X-ray and gamma-ray telescopes and other X-ray equipments.

6. For pumping of X-ray laser. Femtosecond pulses are especially convenient for this purpose, because pulse duration is comparable with lifetime of $\mathrm{K}$ and/or L-shell vacancy in atoms.

7. For control and detecting of nuclear materials in cosmic space, atmosphere, airports, railways and so on. Pulsed PXR monochromatic X-ray beam is very convenient instrument for control of nuclear materials circulation and proliferation in the world. For example, linac-based PXR source of X-ray beam may be launched to cosmic space for remote detecting of nuclear materials in satellites. Scanning of X-ray beam energy around the absorption K-edge may be applied for detection of nuclear materials. 
8. For remote investigations of chemical composition of asteroids and moon, using monochromatic X-ray beam for excitation of characteristic Xrays. Linac-based PXR source of X-ray beam now is the only single perspective source of monochromatic X-ray beam in space. (Synchrotron may not be launched now due to its geometry and size.)

Development and investigations of PXR as a source of powerful polarized $\mathrm{X}$-ray beam will be useful for the above mentioned and other applications. Real crystals have sets of numerous crystallographic planes. As a result, a set of numerous powerful polarized X-ray beams with different energies will be propagated from a crystal, situated in the electron beam, in different directions. Many of those X-ray beams may be utilized simultaneously much like the synchrotron radiations is utilized from different parts of a storage ring. Short X-ray pulses are very convenient for improvement of measurement sensitivities at remote locations. Linear polarization of X-ray beam may be utilized for the same purpose.

We (Z.P.) thank A. Shchagin and ask interested readers to direct their: questions re data, chronology of the experiments and papers in the Former Soviet Union to him. We acknowledge and thank D. Rule for discussions, and ITP Program "New Ideas for Particle Accelerators" for providing partial support for this work - with the National Science Foundation under Grant No. PHY94-07194.

\section{REFERENCES}

1. Fainberg Ya. B. and Khizhnyak N.A., Zh. Ehksp. Teor. Fiz. 32, 883-895 (1957).

2. Ter-Mikaelian M.L., High-Energy Electromagnetic Processes in Condensed Media (Wiley-Interscience, New York, 1972).

3. Baryshevskii V.G., Channeling, Radiation and Reactions in Crystals at High Energies (BGU publ., Minsk, 1982) [in Russian].

4. Garibian G.M. and Yang C., X-ray Transition Radiation (Acad. Sciences of Arm. SSR, Yerevan, 1983) [in Russian].

5. Dialetis D., Phys. Rev. A 17, 1113-1122 (1978).

6. Feranchuk I.D. and Ivashin A.V., J. Physique 46, 1981-1986 (1985).

7. Adishchev Yu.N., Didenko A.N., Mun V.V., Pleshkov G.A., Potylitsin A.P., Tomchakov V.K., Uglov S.R. and Vorobiev S.A., Nucl. Instr. and Meth. B 21, 49-55 (1987).

8. Adishchev Yu.N., Verpinov V.A., Potylitsin A.P., Uglov S.R. and Vorobiev S.A., Nucl. Instr. and Meth. B 44, 130-136 (1989).

9. Shchagin A.V., Pristupa V.I. and Khizhnyak N.A., Phys. Lett. A 148, 485-488 (1990).

10. Morokhovskii V.L. and Shchagin A.V., Zh. Tekh. Fiz. 60, no. 5, 147-150 (1990); In English: Sov. Phys. Tech. Phys. 35, 623-624 (1990). 
11. Shchagin A.V., Pristupa V.I. and Khizhnyak N.A., Proceedings of International Symposium on Radiation of Relativistic Electrons in Periodical Structures, September 6-10, 1993, Tomsk, Russia, p.62-75. See also [16].

12. Shchagin A.V., Pristupa V.I. and Khizhnýak N.A., Nucl. Instr. and Meth. B 99, 277-280 (1995).

13. Fiorito R.B., Rule D.W., Maruyama X.K., DiNova K.L., Evertson S.J., Osborne M.J., Snyder D., Rietdyk H., Piestrup M.A. and Ho A.H., Phys. Rev. Lett. 71, 704-707 (1993).

14. Fiorito R.B., Rule D.W., Piestrup M.A., Qiang Li, Ho A.H. and Maruyama X.K, Nucl. Instr. and Meth. B 79, 758-761 (1993).

15. Fiorito R.B., Rule D.W., Piestrup M.A., Maruyama X.K., Silzer R.M., Skopik D.M. and Shchagin A.V., Phys. Rev. E 51, Part 1, R2759-R2762 (1995).

16. Reviews about recent investigations of PXR properties: A.V. Shchagin, The Future of Accelerator Physics, The Tamura Symposium Proceedings, Austin TX, November 1994, AIP Press, pp.359 - 377; A.V. Shchagin, N.A. Khizhnyak, will be published in October 1996 issue in NIM B; A.V. Shchagin, X.K. Maruyama, Parametric X-rays. Will be published as a chapter in book: Accelerator based atomic physics technique and applications, edited by S.M. Shafroth and J.C. Austin, AIP Press, 1997.

17. Z. Parsa, "Pulsed Parametric X-Radiation", Presentation UCSB/ITP96 Santa Barbara, Report (1996).

18. Z. Parsa, "New Ideas for Particle Accelerators" Program Summary Report, Santa Barbara, Ca. (1997).

19. Z. Parsa, "Pulsed Parametric X-Radiation (PPXR) - A New Alternative to Synchrotron Source", American Physical Society Presentation, APS-APR97 LogNo. 6023, (April 1997).

\section{FIGURE CAPTIONS}

Fig. 1. The experimental setup for generation of powerful, pulsed, monochromatic, polarized x-ray beam. Electron beam 1 is passing through a crystal slab 2. Vector $\vec{g}=<111>$ is the reciprocal lattice vector for the crystallographic planes (111) shown by hatching on $S i$ single crystal target 2. Target 2 is placed in a vacuum 3 -axis goniometer and the angle $\phi$ is the angle of crystal rotation. The PXR reflection 3 has asymmetrical conical form around of Bragg direction 4. The X-ray beam polarized in the plane of figure is going at angle $\theta_{B}+\frac{1}{\gamma}$ to consumer 5 in full cone angle equal to $1 / \gamma$.

Fig. 2. Power $[\mathrm{MW} / \mathrm{sr}]$ of polarized monochromatic X-ray beam as function of photon energy for incident electron beam energy of 50,100, $200 \mathrm{MeV}$. Duration of X-ray beam pulse is $333 \mathrm{fs}$. Charge of electron bunch is $1 \mathrm{nC}$. Angle $\theta$ of X-ray beam generation is in the range 40-170 mrad. Thickness of Si single crystal is $60 \mathrm{mkm}$. 


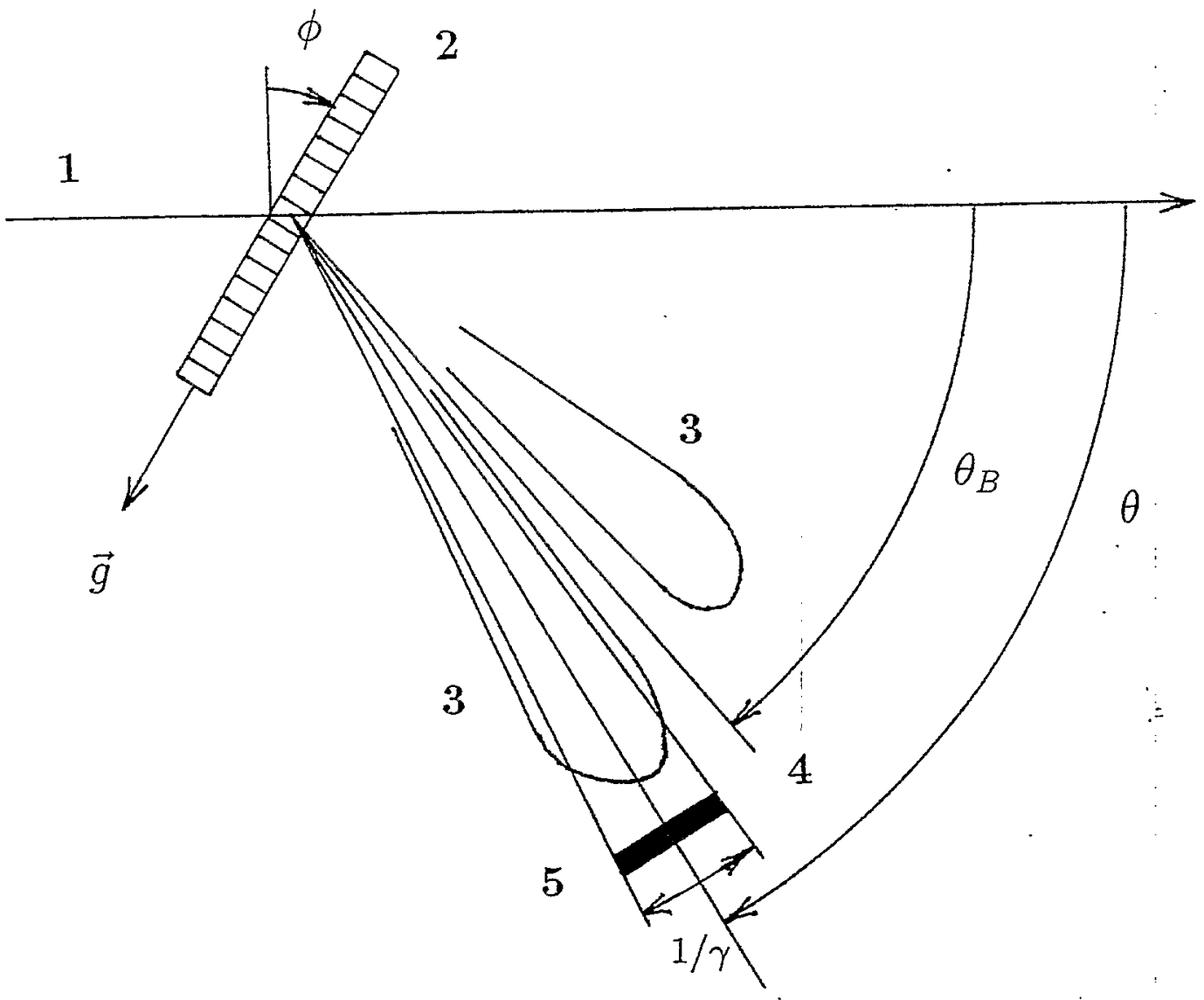

Fig. 1 


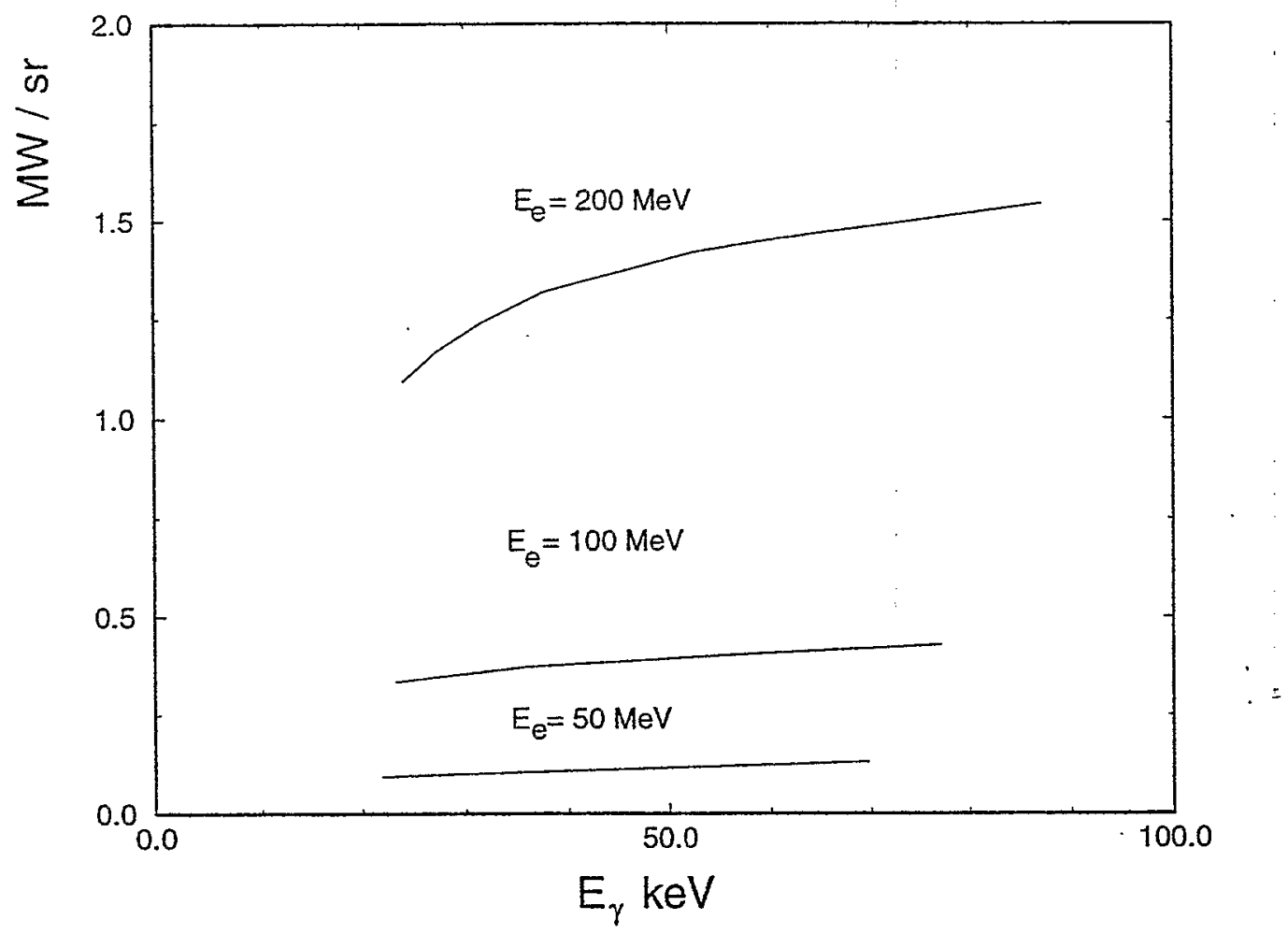

Fig. 2 